\title{
ПРИМЕНЕНИЕ РАСШИРЕННОГО ФИЛЬТРА КАЛМАНА ДЛЯ ИДЕНТИФИКАЦИИ ПАРАМЕТРОВ РАСПРЕДЕЛЕННОЙ ДИНАМИЧЕСКОЙ СИСТЕМЫ
}

\author{
А. В. Копытин, Е. А. Копытина, М. Г. Матвеев \\ Воронежский государственный университет
}

Поступила в редакцию 30.09.2018 г.

\begin{abstract}
Аннотация. Предложен комбинированный метод идентификации уравнений математической физики, описывающих динамику пространственно-распределенных процессов, на основе экспериментальных многомерных временных рядов. Первой составляющей метода является получение МНК-оценок параметров многомерной авторегрессии. Однако, эти оценки оказываются смещенными в силу наличия ошибок в регрессорах. С целью уменьшения указанного смещения в качестве второй составляющей метода применяется расширенный фильтр Калмана. Приводится вычислительный эксперимент, подтверждающий эффективность предложенной методики.

Ключевые слова: пространственно-распределенные процессы, оценка параметров, МНК, многомерная авторегрессия, расширенный фильтр Калмана.

Annotation. A combined method for identifying the equations of mathematical physics describing the dynamics of spatially distributed processes is proposed on the basis of experimental multidimensional time series. The first component of the method is the derivation of OLS estimators of multidimensional autoregression parameters. However, these estimates are biased due to the presence of errors in the regressors. In order to reduce this displacement, a dilated Kalman filter is used as the second component of the method. A computational experiment confirming the effectiveness of the proposed method is given.

Keywords: spatially distributed processes, parameter estimation, LSM, multidimensional autoregression, extended Kalman filter.
\end{abstract}

\section{ВВЕДЕНИЕ}

В настоящее время задачи идентификации параметров динамических систем являются одними из важнейших задач технических, экономических и социальных приложений. Им посвящено большое число работ. При этом большинство работ посвящено стационарным системам с сосредоточенными параметрами (см., например, [1-7]). Значительно меньше работ посвящено идентификации динамических систем и систем с распределенными параметрами, причем в большинстве этих работ рассматриваются приближенные методы, в том числе, основанные на обработке наблюдений многомерных временных рядов в узлах разностных схем, аппроксимиру-

() Копытин А. В., Копытина Е. А., Матвеев М. Г., 2018 ющих соответствующие дифференциальные уравнения [8-10].

Продолжая исследования, начатые в [1113], будем рассматривать широкий класс пространственно-распределенных динамических систем, для которых характерны диффузионные процессы, процессы адвекции или их сочетание. Соответствующее дифференциальное уравнение в частных производных с начальными и граничными условиями имеет следующий общий вид:

$$
\begin{aligned}
& \frac{\partial x}{\partial t}+v \frac{\partial x}{\partial l}=D \frac{\partial^{2} x}{\partial l^{2}}, \\
& x(0, l)=\varphi(l), \\
& x\left(t, l^{\min }\right)=f_{1}(t), \quad x\left(t, l^{\max }\right)=f_{2}(t),
\end{aligned}
$$

где $v$ - скорость адвекции, $D$ - коэффициент диффузии, $l$ - пространственная координата.

Источником информации о поведении системы являются данные натурных измере- 
ний переменной $x_{i}^{t}$ с погрешностью $\varepsilon_{i}^{t}$ в виде нормального «белого шума» $-y_{i}^{t}=x_{i}^{t}+\varepsilon_{i}^{t} \mathrm{c}$ математическим ожиданием $M\left(\varepsilon_{i}\right)=0$ и $\operatorname{cov}\left(\varepsilon_{i}, \varepsilon_{j}\right)=0$, в последовательные моменты времени $t=0,1, \ldots, n$ в узлах одномерной пространственной регулярной сетки $i=0,1, \ldots, m$, т. е. многомерный временной ряд. Рассмотрение одномерной сетки ничем не ограничивает дальнейшие исследования, зато позволяет избежать громоздких построений, характерных для плоских и объемных пространств.

Задача заключается в верификации процессов конвективной диффузии на основе анализа многомерных временных рядов и разработке алгоритмов параметрической идентификации механистической модели с постоянными коэффициентами по наблюдаемым значениям $y_{i}^{t}$.

Для решения задачи составим явную четырехточечную разностную схему для уравнения (1):

$$
\begin{gathered}
\frac{x_{i}^{t+1}-x_{i}^{t}}{\Delta t}+v \frac{x_{i+1}^{t}-x_{i-1}^{t}}{2 \Delta l}=D \frac{x_{i+1}^{t}-2 x_{i}^{t}+x_{i-1}^{t}}{\Delta l^{2}}, \\
x_{i}^{t+1}=\left(b_{1}+b_{2}\right) x_{i-1}^{t}+\left(1-2 b_{2}\right) x_{i}^{t}+\left(b_{2}-b_{1}\right) x_{i+1}^{t} ; \\
b_{1}=\frac{v \Delta t}{2 \Delta l} ; \quad b_{2}=\frac{D \Delta t}{\Delta l^{2}} .
\end{gathered}
$$

Уравнения (2) можно записать в приведенном виде

$$
x_{i}^{t+1}=a_{1} x_{i-1}^{t}+a_{2} x_{i}^{t}+a_{3} x_{i+1}^{t}, \quad t=0,1, \ldots, n,
$$

где $a_{i}$ - оцениваемые регрессионные параметры, связанные с параметрами $b_{1}$ и $b_{2}$ следующими соотношениями

$$
\left\{\begin{array}{l}
b_{1}+b_{2}=a_{1}, \\
1-2 b_{2}=a_{2}, \\
b_{2}-b_{1}=a_{3} .
\end{array}\right.
$$

Из выражения (2) следует, что параметры $a_{i}$ удовлетворяют следующему равенству

$$
a_{1}+a_{2}+a_{3}=1
$$

Для вычисления оценок параметров $a_{i}$ выполним следующие преобразования переменных разностной схемы (3).

В матричной форме выражение (3) принимает вид

$$
\mathbf{x}=\mathbf{X a},
$$

где

$$
\mathbf{x}=\left(\begin{array}{c}
x_{i}^{1} \\
x_{i}^{2} \\
\vdots \\
x_{i}^{n}
\end{array}\right), \quad \mathbf{X}=\left(\begin{array}{ccc}
x_{i-1}^{0} & x_{i}^{0} & x_{i+1}^{0} \\
x_{i-1}^{1} & x_{i}^{1} & x_{i+1}^{1} \\
\vdots & \vdots & \vdots \\
x_{i-1}^{n-1} & x_{i}^{n-1} & x_{i+1}^{n-1}
\end{array}\right), \mathbf{a}=\left(\begin{array}{c}
a_{1} \\
a_{2} \\
a_{3}
\end{array}\right)
$$

Обозначим $\mathbf{y}=\mathbf{x}+\boldsymbol{\varepsilon}, \mathbf{Y}=\mathbf{X}+\mathbf{E}$, где $\boldsymbol{\varepsilon}$ и $\mathbf{E}$ - соответственно вектор и матрица ошибок измерений, т. е.

$$
\boldsymbol{\varepsilon}=\left(\begin{array}{c}
\varepsilon_{i}^{1} \\
\varepsilon_{i}^{2} \\
\vdots \\
\varepsilon_{i}^{n}
\end{array}\right), \quad \mathbf{E}=\left(\begin{array}{ccc}
\varepsilon_{i-1}^{0} & \varepsilon_{i}^{0} & \varepsilon_{i+1}^{0} \\
\varepsilon_{i-1}^{1} & \varepsilon_{i}^{1} & \varepsilon_{i+1}^{1} \\
\vdots & \vdots & \vdots \\
\varepsilon_{i-1}^{n-1} & \varepsilon_{i}^{n-1} & \varepsilon_{i+1}^{n-1}
\end{array}\right)
$$

В новых обозначениях уравнение (6) имеет вид

$$
\mathbf{y}=\mathbf{Y a}+(\boldsymbol{\varepsilon}-\mathbf{E a})
$$

Уравнение (7) позволяет записать МНК-оценку $\hat{a}=\left(\hat{a}_{1} ; \hat{a}_{2} ; \hat{a}_{3}\right)^{T}$ вектора параметров $\mathbf{a}$

$$
\hat{\mathbf{a}}=\left(\mathbf{Y}^{T} \mathbf{Y}\right)^{-1} \mathbf{Y}^{T} \mathbf{y}=\mathbf{a}+\left(\mathbf{Y}^{T} \mathbf{Y}\right)^{-1} \mathbf{Y}^{T}(\boldsymbol{\varepsilon}-\mathbf{E a}) .
$$

Найдем математическое ожидание левой и правой части выражения (8)

$$
M(\hat{a})=a+M\left[\left(Y^{T} Y\right)^{-1} Y^{T}(\varepsilon-E a)\right] .
$$

Как видно из полученного равенства (9) математическое ожидание оценки $\hat{a}$ отличается от истинного вектора $a$ на величину $M\left[\left(Y^{T} Y\right)^{-1} Y^{T}(\varepsilon-E a)\right] \neq 0$, которая интерпретируется как смещение компонент вектора оценки.

Наличие смещения может существенно повлиять на оценку параметров $b_{1}$ и $b_{2}$, определяемых на основе системы уравнений (4). Дело в том, что теперь в системе (4) вместо параметров $a$ будут фигурировать случайные значения компонент вектора оценок этих параметров. В этом случае для вычисления оценок $\hat{b}=\left(\hat{b}_{1}, \hat{b}_{2}\right)$ предлагается следующая методика. Например, из первого и третьего уравнений системы (4) вычисляются оценки $\hat{b}=\left(\hat{b}_{1}, \hat{b}_{2}\right)=\left(\hat{a}_{1}-\hat{a}_{3}, \hat{a}_{1}+\hat{a}_{3}\right)^{T} / 2$, которые затем проверяются на возможность принятия нулевой гипотезы о выполнении равенств $\hat{a}_{1}+\hat{a}_{2}+\hat{a}_{3}=1$ и $\hat{a}_{2}+2 \hat{b}_{2}=1$. Если указанные равенства можно принять, например, на $5 \%$ уровне значимости, полученные оценки рассматриваются как удовлетворяющие системе (4). Наличие смещения оценки вектора $a$ увеличивает вероятность отклонения нулевой 


\section{А. В. Копьттин, Е. А. Копьытина, М. Г. Матвеев}

гипотезы, что снижает эффективность применения МНК-оценок даже при малых погрешностях наблюдений и соответственно малых стандартных ошибках оценок параметров.

Повышения точности решения задачи верификации процессов уравнения (1) с использованием МНК-оценок параметров авторегрессии (3) можно достичь на основе комбинирования методов идентификации. Такой подход получил широкое распространение в различных предметных областях (см., например, $[14,15])$. Комбинирование дает возможность компенсировать недостатки одних методов при помощи других и направлено на повышение качества параметрической идентификации, как одного из главных критериев эффективности модели. Мы предлагаем для решения задачи верификации использовать комбинацию МНК-оценок и фильтра Калмана. МНК-оценки дают начальную точку в пространстве поиска для реализации рекурсивного алгоритма Калмана, что очевидно лучше, чем произвольный выбор такой точки. Исследованию эффективности комбинации МНК-оценок и фильтра Калмана посвящена предлагаемая работа.

\section{ФИЛЬТР КАЛМАНА С ВЫБОРОМ НАЧАЛЬНОГО ЗНАЧЕНИЯ ОЦЕНКИ}

Как известно фильтр Калмана представляет собой рекурсивный алгоритм оптимального оценивания неизвестного состояния линейной динамической системы по зашумленным измерениям в дискретные моменты времени. Если система оказывается нелинейной, обычно используется процедура линеаризации. Полученный таким образом фильтр называется расширенным фильтром Калмана.

Рассматриваемая нами линейная динамическая модель может быть записана в пространстве состояний следующим образом

$$
\left\{\begin{array}{l}
\mathbf{x}_{k+1}=\mathbf{A}(\mathbf{b}) \mathbf{x}_{k}+\mathbf{u}_{k}+\boldsymbol{\xi}_{k}, \\
\mathbf{y}_{k}=\mathbf{H} \mathbf{x}_{k}+\boldsymbol{\eta}_{k},
\end{array}\right.
$$

где $\mathbf{x}_{k}=\left(x_{i-2}^{k}, x_{i-1}^{k}, x_{i}^{k}, x_{i+1}^{k}, x_{i+2}^{k}\right)^{T}-$ вектор состояния, $\mathbf{A}(\mathbf{b})$ - матрица эволюции процесса, зависящая от постоянного вектора $\mathbf{b}=\left(b_{1}, b_{2}\right)^{T}$, $\mathbf{u}_{k}=\left(y_{i-2}^{k+1}, 0,0,0, y_{i+2}^{k+1}\right)^{T}$ интерпретируется в терминах фильтра Калмана как вектор управляющих воздействий, $\boldsymbol{\xi}_{k}=\left(\varepsilon_{i-2}^{k+1}, 0,0,0, \varepsilon_{i+2}^{k+1}\right)^{T}-$ шум процесса, $\mathbf{y}_{k}=\left(y_{i-1}^{k}, y_{i}^{k}, y_{i+1}^{k}\right)^{T}$ - вектор измерений, $\mathbf{H}$ - матрица измерений, $\eta_{k}=\left(\varepsilon_{i-1}^{k}, \varepsilon_{i}^{k}, \varepsilon_{i+1}^{k}\right)^{T}$ - измерительный шум.

$$
\begin{gathered}
\mathbf{A}(\mathbf{b})=\left(\begin{array}{ccccc}
0 & 0 & 0 & 0 & 0 \\
b_{1}+b_{2} & 1-2 b_{2} & b_{2}-b_{1} & 0 & 0 \\
0 & b_{1}+b_{2} & 1-2 b_{2} & b_{2}-b_{1} & 0 \\
0 & 0 & b_{1}+b_{2} & 1-2 b_{2} & b_{2}-b_{1} \\
0 & 0 & 0 & 0 & 0
\end{array}\right), \\
\mathbf{H}=\left(\begin{array}{ccccccc}
0 & 1 & 0 & 0 & 0 & 0 & 0 \\
0 & 0 & 1 & 0 & 0 & 0 & 0 \\
0 & 0 & 0 & 1 & 0 & 0 & 0
\end{array}\right) .
\end{gathered}
$$

Поскольку $\mathbf{b}$ - постоянный вектор, вполне естественно положить

$$
\mathbf{b}_{k+1}=\mathbf{b}_{k} \text {. }
$$

Тогда система (10) вместе с предположением (11) может быть переформулирована как нелинейная модель

$$
\left\{\begin{array}{l}
\left(\begin{array}{l}
\mathbf{x}_{k+1} \\
\mathbf{b}_{k+1}
\end{array}\right)=\left(\begin{array}{c}
\mathbf{A}\left(\mathbf{b}_{k}\right) \mathbf{x}_{k} \\
\mathbf{b}_{k}
\end{array}\right)+\left(\begin{array}{c}
\mathbf{u}_{k} \\
0
\end{array}\right)+\left(\begin{array}{c}
\boldsymbol{\xi}_{k} \\
0
\end{array}\right), \\
\mathbf{y}_{k}=\left(\begin{array}{ll}
\mathbf{H} & 0
\end{array}\right)\left(\begin{array}{l}
\mathbf{x}_{k} \\
\mathbf{b}_{k}
\end{array}\right)+\boldsymbol{\eta}_{k},
\end{array}\right.
$$

и расширенный фильтр Калмана может быть применен для оценки вектора состояния, содержащего $\mathbf{b}_{k}$ в качестве своих компонент. Однако, для этого нам необходимо иметь оценки матриц ковариации $\mathbf{Q}=\operatorname{cov}\left(\boldsymbol{\xi}_{k}\right)$ и $\mathbf{R}=\operatorname{cov}\left(\boldsymbol{\eta}_{k}\right)$, начальную оценку вектора состояния $\left(\begin{array}{c}\hat{\mathbf{x}}_{0 \mid 0} \\ \hat{\mathbf{b}}_{0 \mid 0}\end{array}\right)$ и оценку ковариационной матрицы $\mathbf{P}_{0 \mid 0}$ начальной оценки вектора состояния.

Пусть $\mathbf{e}=\mathbf{y}-\mathbf{Y a ̂}$ - вектор остатков регрессии (7). В качестве оценки дисперсии измерительной погрешности $\varepsilon_{i}^{t}$ возьмем величину $v_{0}=\mathbf{e}^{T} \mathbf{e} /(n-3) /\left(1+\hat{\mathbf{a}}^{T} \hat{\mathbf{a}}\right)$. Тогда

$$
\mathbf{Q}=\operatorname{diag}\left\{v_{0}, 0,0,0, v_{0}, 0,0\right\}, \mathbf{R}=\operatorname{diag}\left\{v_{0}, v_{0}, v_{0}\right\},
$$

где diag обозначает диагональную матрицу.

В качестве оценки $\hat{\mathbf{x}}_{00}$ естественно взять вектор $\left(y_{i-2}^{0}, y_{i-1}^{0}, y_{i}^{0}, y_{i+1}^{0}, y_{i+2}^{0}\right)^{T}$, а в качестве оценки $\hat{\mathbf{b}}_{0 \mid 0}$ МНК-оценку $\mathbf{b}=\left(\hat{a}_{1}-\hat{a}_{3}, \hat{a}_{1}+\hat{a}_{3}\right)^{T} / 2$. Оценка ковариационной матрицы вектора МНК-оценок $\hat{\mathbf{a}}$ имеет вид $\mathbf{V}_{\mathbf{a}}=\left(v_{i j}\right)=\mathbf{e}^{T} \mathbf{e} /(n-3)\left(\mathbf{Y}^{T} \mathbf{Y}\right)^{-1}$. 
Тогда в качестве оценки ковариационной матрицы вектора $\hat{\mathbf{b}}$ возьмем следующую матрицу $\mathbf{V}_{\mathbf{b}}$

$$
\mathbf{V}_{\mathbf{b}}=\left(\begin{array}{cc}
1 / 2 & -1 / 2 \\
1 / 2 & 1 / 2
\end{array}\right)\left(\begin{array}{cc}
v_{11} & v_{13} \\
v_{31} & v_{33}
\end{array}\right)\left(\begin{array}{cc}
1 / 2 & 1 / 2 \\
-1 / 2 & 1 / 2
\end{array}\right),
$$

и матрица $\mathbf{P}_{0 \mid 0}$ примет вид

$$
\mathbf{P}_{0 \mid 0}=\left(\begin{array}{cc}
\operatorname{diag}\left\{v_{0}, v_{0}, v_{0}, v_{0}, v_{0}\right\} & 0 \\
0 & \mathbf{V}_{\mathbf{b}}
\end{array}\right) .
$$

Алгоритм расширенного фильтра Калмана в нашем случае выглядит следующим образом (см. [16])

$$
\begin{aligned}
& \int\left(\begin{array}{c}
\hat{\mathbf{x}}_{0 \mid 0} \\
\hat{\mathbf{b}}_{0 \mid 0}
\end{array}\right)=\left(\begin{array}{c}
\left(y_{i-2}^{0}, y_{i-1}^{0}, y_{i}^{0}, y_{i+1}^{0}, y_{i+2}^{0}\right)^{T} \\
\hat{\mathbf{b}}
\end{array}\right), \\
& \mathbf{P}_{0 \mid 0}=\left(\begin{array}{cc}
\operatorname{diag}\left\{v_{0}, v_{0}, v_{0}, v_{0}, v_{0}\right\} & 0 \\
0 & \mathbf{V}_{\mathbf{b}}
\end{array}\right), \\
& \text { Для } k=1,2, \ldots, n \text {, } \\
& \left(\begin{array}{c}
\hat{\mathbf{x}}_{k \mid k-1} \\
\hat{\mathbf{b}}_{k \mid k-1}
\end{array}\right)=\left(\begin{array}{c}
\mathbf{A}\left(\hat{\mathbf{b}}_{k-1 \mid k-1}\right) \hat{\mathbf{x}}_{k-1 \mid k-1}+\mathbf{u}_{k} \\
\hat{\mathbf{b}}_{k-1 \mid k-1}
\end{array}\right) \text {, } \\
& \left\{\mathbf{P}_{k \mid k-1}=\left(\begin{array}{cc}
\mathbf{A}\left(\hat{\mathbf{b}}_{k-1 \mid k-1}\right) & \frac{\partial}{\partial \mathbf{b}}\left(\mathbf{A}\left(\hat{\mathbf{b}}_{k-1 \mid k-1}\right) \hat{\mathbf{x}}_{k-1 \mid k-1}\right) \\
0 & \mathbf{I}
\end{array}\right) \times\right. \\
& \times \mathbf{P}_{k-1 \mid k-1}\left(\begin{array}{cc}
\mathbf{A}\left(\hat{\mathbf{b}}_{k-1 \mid k-1}\right) & \frac{\partial}{\partial \mathbf{b}}\left(\mathbf{A}\left(\hat{\mathbf{b}}_{k-1 \mid k-1}\right) \hat{\mathbf{x}}_{k-1 \mid k-1}\right) \\
0 & \mathbf{I}
\end{array}\right)^{T} \\
& +\left(\begin{array}{ll}
\mathbf{Q} & 0 \\
0 & 0
\end{array}\right) \\
& \mathbf{K}_{k}=\mathbf{P}_{k \mid k-1}\left(\begin{array}{lll}
\mathbf{H} & 0
\end{array}\right)^{T}\left(\left(\begin{array}{lll}
\mathbf{H} & 0
\end{array}\right) \mathbf{P}_{k \mid k-1}\left(\begin{array}{ll}
\mathbf{H} & 0
\end{array}\right)^{T}+\mathbf{R}\right)^{-1}, \\
& \mathbf{P}_{k \mid k}=\left(\mathbf{I}-K_{k}\left(\begin{array}{ll}
\mathbf{H} & 0
\end{array}\right)\right) \mathbf{P}_{k \mid k-1}, \\
& \left(\begin{array}{c}
\hat{\mathbf{x}}_{k \mid k} \\
\hat{\mathbf{b}}_{k \mid k}
\end{array}\right)=\left(\begin{array}{c}
\hat{\mathbf{x}}_{k \mid k-1} \\
\hat{\mathbf{b}}_{k \mid k-1}
\end{array}\right)+\mathbf{K}_{k}\left(\mathbf{y}_{k}-\mathbf{H} \hat{\mathbf{x}}_{k \mid k-1}\right) \text {. }
\end{aligned}
$$

В качестве итоговой оценки вектора параметров b будем использовать последнее значение оценки $\hat{\mathbf{b}}_{n \mid n}$, полученное при $k=n$.

\section{РЕЗУЛЬТАТЫ ВЫЧИСЛИТЕЛЬНОГО ЭКСПЕРИМЕНТА}

Для проведения исследований удобно воспользоваться данными модельного эксперимента. Для этого необходимо найти решение исходного дифференциального уравне- ния (1) с заданными значениями параметров $v$ и $D$, которые легко пересчитываются в параметры $b_{1}$ и $b_{2}$ разностной схемы. Затем выполнить регулярную дискретизацию полученного решения и добавить погрешность $\varepsilon$ в виде «белого шума» с различной интенсивностью. Полученные статистические данные будут использованы для получения оценок $\hat{b}_{1}$ и $\hat{b}_{2}$ параметров разностной схемы. Таким образом, модельный эксперимент позволяет провести наглядное сравнение исходных значений параметров и их оценок при различных методах получения оценок и различных интенсивностях помехи.

Рассмотрим задачу (1) на отрезке [1;3] с такими функциями $\varphi, f_{1}$ и $f_{2}$, что ее решение имеет вид

$$
\begin{aligned}
& x(t, l)=\exp \left(\frac{v}{2 D}\left(l-\frac{v t}{2}\right)\right)(\exp (-D t) \sin (l)+ \\
& +\exp (-4 D t) \sin (2 l)+\exp (-9 D t) \sin (3 l))
\end{aligned}
$$

Пусть значения параметров $v$ и $D$ равны соответственно 2 и 3, шаг по пространственной координате $\Delta l=0,1$, шаг по времени $\Delta t=\Delta l^{2} /(4 D)$, что соответствует условиям Куранта для обеспечения устойчивости аппроксимирующей разностной схемы; $n=1000$.

Далее к значениям $x_{i}^{t}$ решения (14) в узлах пространственно-временной сетки добавим смоделированную с помощью генератора случайных чисел погрешность в виде нормального «белого шума» с дисперсией $\sigma$ и по полученным значениям $y_{i}^{t}$ найдем сначала МНК-оценки $\hat{a}$ параметров разностной схемы (3) и оценки $\hat{b}_{1}$ и $\hat{b}_{2}$ с помощью системы (4) и предложенной методики. Затем, на заданном уровне значимости, проверяются статистические гипотезы $\hat{a}_{1}+\hat{a}_{2}+\hat{a}_{3}=1$ и $\hat{a}_{2}+2 \hat{b}_{2}=1$, принятие которых дает основание считать оценки $\hat{b}_{1}$ и $\hat{b}_{2}$ адекватными. На основе полученных оценок разностной схемы (2) вычисляются оценки параметров дифференциального уравнения - $v$ и $D$. Вычислительный эксперимент направлен на сравнительный анализ трех подходов к оценке параметров дифференциального уравнения: на основе обыкновенного МНК; с помощью фильтра Калмана, используя произвольный выбор начальной оценки вектора $\mathbf{b}$, а также 


\section{А. В. Копытин, Е. А. Копьгтина, М. Г. Матвеев}

подхода, использующего последовательную комбинацию МНК и фильтра Калмана.

Очевидно, что результаты параметрической идентификации могут существенно зависеть от интенсивности «белого шума», задаваемой стандартным отклонением случайной погрешности наблюдений $-\sigma$. Для проведения эксперимента были выбраны три уровня погрешности: $\sigma=0,001 ; \sigma=0,005$; $\sigma=0,01$. Эксперимент с оценками на основе фильтра Калмана проводился при значении начальной оценки $\hat{\mathbf{b}}_{0 \mid 0}=(0 ; 0)^{T}$ вектора b. Результаты идентификации представлены в табл. 1.

Сравнение качества идентификации. по статистическому критерию

\begin{tabular}{|c|c|c|c|c|c|c|}
\hline \multirow{2}{*}{$\sigma$} & \multicolumn{6}{|c|}{ МАРЕ $^{\text {а }}$} \\
\cline { 2 - 7 } & \multicolumn{2}{|c|}{ МНК } & \multicolumn{2}{c|}{$\begin{array}{c}\text { Фильтр } \\
\text { Калмана }\end{array}$} & $\begin{array}{c}\text { Комби- } \\
\text { ниро- } \\
\text { ванный } \\
\text { метод }\end{array}$ \\
\cline { 2 - 7 } & $v$ & $D$ & $v$ & $D$ & $v$ & $D$ \\
\hline 0,001 & 7,52 & 1,02 & 3,17 & 2,74 & 0,95 & 0,3 \\
\hline 0,005 & 96,3 & 16,3 & 11,11 & 4,58 & 4,21 & 0,67 \\
\hline 0,01 & 158,93 & 26,34 & 32,2 & 8,36 & 6,82 & 1,19 \\
\hline \multicolumn{7}{|c}{ аредня абсолютная ошибка в процентах }
\end{tabular}

Данные табл. 1 показывают, что при небольшой погрешности $(\sigma=0,001)$ средняя абсолютная ошибка оценки параметров $v$ и $D$ различается при использовании МНК и фильтра Калмана не значительно. Комбинированный метод, даже при небольшой погрешности, показывает уменьшение средней абсолютной ошибки на порядок по сравнению с МНК и фильтром Калмана. Увеличение погрешности, сначала в пять раз, а затем на порядок, больше всего сказывается на оценках МНК. При удачном выборе начальной оценки несколько более устойчив к помехам фильтр Калмана. Лучше всего на возрастание помехи реагирует комбинированный метод, скорость роста абсолютной ошибки оценок меньше скорости роста помехи.

Модельный эксперимент повторялся по каждому методу получения оценок и при каждом уровне интенсивности помехи повто- рялся многократно $(m=100)$, что позволило получить среднеарифметические значения оценок параметров $v$ и $D$. Средние значения оценок можно рассматривать как хорошее приближение к их математическому ожиданию. В этом случае разницу между средним значением оценки и ее истинным значением можно принять за величину смещения. Результаты сравнения средних значений исследуемых оценок при различных интенсивностях помех получаются на основании данных табл. 2. Напомним, что модельный эксперимент проводился с известными значениями параметров $v=2$ и $D=3$. Поскольку рассматриваются средние значения оценок, можно считать, что их отклонения от истинных значений можно интерпретировать как величину смещения.

Таблица 2

Средние значения оченок параметров

\begin{tabular}{|c|c|c|c|c|c|c|}
\hline \multirow{2}{*}{$\sigma$} & \multicolumn{2}{|c|}{ МНК } & \multicolumn{2}{c|}{$\begin{array}{c}\text { Фильтр } \\
\text { Калмана }\end{array}$} & \multicolumn{2}{c|}{$\begin{array}{c}\text { Комбини- } \\
\text { рованный } \\
\text { метод }\end{array}$} \\
\cline { 2 - 7 } & $v$ & $D$ & $v$ & $D$ & $v$ & $D$ \\
\hline 0,001 & 2,14 & 3,03 & 1,94 & 2,92 & 1,98 & 2,99 \\
\hline 0,005 & 4 & 3,49 & 1,78 & 2,86 & 1,97 & 2,98 \\
\hline 0,01 & 5,03 & 3,79 & 1,36 & 2,75 & 1,94 & 2,97 \\
\hline
\end{tabular}

Сравнение средних значений с истинными значениями параметров показывает, что величина смещения оценок МНК и фильтра Калмана с произвольным выбором начальной оценки существенна даже при небольших погрешностях $(\sigma=0,001)$ и возрастает с ростом погрешности. При этом смещение оценок комбинированного метода несущественно и незначительно возрастает с ростом погрешности. Отсюда следует вывод о зависимости величины смещения от выбора начального значения оценки, что подтверждает целесообразность предложенной комбинации методов.

\section{ЗАКЛЮЧЕНИЕ}

Результаты проведенных экспериментов показывают, что использование для оценки параметров дифференциального уравнения 
Применение расширенного фильтра Калмана для идентификаџии параметров ...

(1) метода наименьших квадратов может привести к существенным искажениям истинных значений параметров в условиях высокого уровня погрешностей наблюдений за многомерными временными рядами в узлах разностной схемы. Автономное использования для идентификации фильтра Калмана также не может обеспечить приемлемого качества оценок параметров дифференциального уравнения. Качество оценок будет зависеть от выбора начального приближения рекурсивной процедуры оценивания.

Предложенное комбинирование МНК-оценок и оценок фильтра Калмана, как показывают результаты табл. 1 и 2 существенно улучшает качество оценки, за счет рационального выбора начальных значений начального приближения рекурсивной процедуры фильтра Калмана в форме смещенных МНК-оценок.

Оценки, приведенные в табл. 1 и 2 представляют собой результаты усреднения соответствующих выборочных оценок. Это обосновывает интерпретацию разности истинных значений оценок и их средних значений как величину смещения. Анализ варьирования величины смещения при использовании различных подходов к оцениванию при изменении уровня погрешности наблюдений позволяют сделать следующие выводы:

- смещение МНК-оценок и оценок, полученных с помощью фильтра Калмана, зависит от величины погрешности наблюдений; с ростом погрешности наблюдается рост смещения;

- скорость роста смещения оценок фильтра Калмана можно уменьшить так как она зависит от выбора начального значения оценки, удачный выбор обеспечивает меньшую скорость роста смещения;

- предложенный комбинированный метод оценки обеспечивает результат с наименьшим смещением.

\section{СПИСОК ЛИТЕРАТУРЫ}

1. A Bayesian approach to parameter estimation in HIV dynamical models / H. Putter [и др.] // Statistics in Medicine. - 2002. - Vol.21. P. 2199-2214.

2. Huang, Y. Hierarchical Bayesian methods for estimation of parameters in a longitudinal HIV dynamic system / Y. Huang, D. Liu, H. Wu // Biometrics. - 2006. - Vol. 62. - P. 413-423.

3. Huang, Y. A Bayesian approach for estimating antiviral efficacy in HIV dynamic models / Y. Huang, H. Wu // Journal of Applied Statistics. - 2006. - Vol. 33. - P. 155-174.

4. Parameter estimation for differential equations: a generalized smoothing approach (with discussion) / J. O. Ramsay [и др.] // Journal of the Royal Statistical Society. Series B. - 2007. Vol. 69. - P. 741-796.

5. Liang, H. Parameter estimation for differential equation models using a framework of measurement error in regression models / H. Liang, $\mathrm{H}$. Wu // Journal of the American Statistical Association. - 2008. - Vol. 103. - P. 1570-1583.

6. Chen, J. Efficient local estimation for time-varying coefficients in deterministic dynamic models with applications to HIV-1 dynamics / J. Chen, H. Wu // Journal of the American Statistical Association. - 2008. - Vol. 103. - P. 369-384.

7. Cao, J. Penalized nonlinear least squares estimation of time-varying parameters in ordinary differential equations / J. Cao, J. Z. Huang, $\mathrm{H}$. Wu // Journal of Computational and Graphical Statistics. - 2012. - Vol. 21. - P. 42-56.

8. Muller, T. Fitting parameters in partial differential equations from partially observed noisy data / T. Muller, J. Timmer // Physical Review, D. - 2002. - Vol. 171. - P. 1-7.

9. Muller, T. Parameter identification techniques for partial differential equations / T. Muller, J. Timmer // International Journal of Bifurcation and Chaos. - 2004. - Vol. 14. - P. 2053-2060.

10. Parameter estimation of partial differential equation models / X. Xun [и др.] // Journal of the American Statistical Association. - 2013. Vol. 108. - P. 1009-1020.

11. Modeling of nonstationary distributed processes on the basis of multidimensional time 
series / M. G. Matveev [и др.] // Procedia Engineering. - 2017. - Vol. 201. - P. 511-516.

12. Verification of the convective diffusion process based on the analysis of multidimensional time series / M. G. Matveev [и др.] // CEUR Workshop Proceedings. - 2017. - Vol. 2022. P. 354-358.

13. Матвеев, М. Г. Двухшаговый метод идентификации распределенной динамической системы: электронный ресурс / М. Г. Матвеев, А. В. Копытин, Е. А. Копытина // Информатика: проблемы, методология, технологии: сборник материалов 18-й международной научно-методической конферен-

Копытин А. В. - канд. физ.-мат. наук, доцент кафедры информационных технологий управления, факультет компьютерных наук, Воронежский государственный университет. E-mail: alexkopytin@gmail.com

Копытина Е. А. - ассистент кафедры информационных технологий управления, факультет компьютерных наук, Воронежский государственный университет.

E-mail: zhemkaterina@yandex.ru

Матвеев М. Г. - д-р техн. наук, профессор, заведующий кафедрой информационных технологий управления, факультет компьютерных наук, Воронежский государственного университет.

E-mail:mgmatveev@yandex.ru ции, Воронеж 8-9 февраля 2018 г.: в 7 т. - Воронеж, 2018 . - T. 5. - C. 185-191.

14. Fogler, H. R. A pattern recognition model for forecasting / H. R. Fogler // Management science. - 1974. - Vol. 20. - P. 1178-1189.

15. Day-ahead electricity price forecasting using the wavelet transform and ARIMA models / A. J. Conejo [и др.] // IEEE transaction on power systems. - 2005. - Vol. 20. - P. 1035-1042.

16. Chui, C. K. Kalman filtering with real-time applications / C. K. Chui, G. Chen. - 3-е изд., перераб. и доп. - Berlin: Springer-Verlag Berlin Heidelberg, 2009. - 241 p.

Kopytin A. V. - Ph. D. of Physical and Mathematical Sciences, Associate Professor, Department of Information Technologies in Management, Computer Sciences Faculty, Voronezh State University. E-mail: alexkopytin@gmail.com

Kopytina E. A. - Postgraduate Student, Department of Information Technologies in Management, Computer Sciences Faculty, Voronezh State University.

E-mail: zhemkaterina@yandex.ru

Matveev M. G. - Ph. D. of Technical Sciences, Full Professor, Head of Department of Information Technologies in Management, Computer Sciences Faculty, Voronezh State University. E-mail: mgmatveev@yandex.ru 